\title{
Individualized sleep promotion in acute care hospitals: managing specific factors that affect patient sleep
}

\author{
Lichuan Ye ${ }^{1}$, Patricia C. Dykes ${ }^{2}$ \\ ${ }^{1}$ Bouvé College of Health Sciences School of Nursing, Northeastern University, Boston, USA; ${ }^{2}$ Department of Medicine, Brigham and Women's \\ Hospital, Boston, USA \\ Contributions: (I) Conception and design: All authors; (II) Administrative support: L Ye; (III) Provision of study materials or patients: All authors; \\ (IV) Collection and assembly of data: L Ye; (V) Data analysis and interpretation: L Ye; (VI) Manuscript writing: All authors; (VII) Final approval of \\ manuscript: All authors. \\ Correspondence to: Lichuan Ye, PhD, RN. Associate Professor, Bouvé College of Health Sciences School of Nursing, Northeastern University, 207c \\ Robinson Hall, 360 Huntington Avenue, Boston, MA 02115, USA. Email: 1.ye@northeastern.edu.
}

Background: Improving sleep for hospitalized patients is an essential clinical need. Compared to the traditional "one-size-fits-all" interventions designed by clinicians for all patients, an individualized strategy engaging patients to identify salient sleep disruptors and seek their input how to address these disruptors are more likely to succeed. Followed by our work of developing the Factors Affecting Inpatient Sleep (FAIS) scale, in this report we developed a set of icons illustrating 14 common sleep disruptors on the FAIS scale, and proposed behavioral sleep promotion tips addressing each sleep disruptor. The set of icons and sleep promotion tips were built into the mobile health (mHealth) tool SLEEPKit, which was the start of our endeavor using mHealth technology to support individualized sleep promotion.

Methods: A participatory iterative approach including feedbacks from patients, family members, and clinicians was used to develop and refine the icons and sleep promotion tips. Focus groups were used to inform the initial development and to brainstorm for the refinement of the icons. Individual interviews with patients and clinicians were conducted to validate each version of the icons using a standardized Content Validity Index (CVI) on a 4-point Likert scale, and offered comments and suggestions for improvement. Strategies of sleep promotion were first identified by the literature review, and then enriched by the summary of data from our previous work. Focus groups were conducted in order to learn empirically if the sleep promotion tips on the current version were acceptable to both patients and clinicians, and how they could be improved.

Results: Six focus groups were conducted and achieved saturation in suggestions for improvement. A total of 5 patients and 3 family members who served on the Patient/ Family Advisory Council, and 42 nurses who served on the interdisciplinary professional practice committees participated in the focus groups. A total of 75 patients and 50 clinicians offered individual feedback and the CVI test for the icons. Successive two or four phases of iterative icon evaluation and refinement were carried out until the average CVI ratings for each icon achieved 3 and above. The sleep promotion tips were created to demonstrate a collaborative effort between patients and clinicians. To empower patients for bedside communication related to their sleep, examples of communication starters ("Try saying") were included in the tips for patients.

Conclusions: By using the participatory iterative approach, these icons for common sleep disturbing factors were understandable by both patients and clinicians, and the sleep promotion tips were perceived to be feasible and effective in the acute care hospital setting. This work moved the individualized sleep promotion forward, and supported the development of a novel mHealth tool for inpatient sleep promotion tailored to individual patient's needs.

Keywords: Sleep promotion; acute care; hospitalized patients; mobile health (mHealth)

Received: 10 January 2020; Accepted: 03 June 2020; Published: 20 April 2021.

doi: $10.21037 /$ mhealth-20-31

View this article at: http://dx.doi.org/10.21037/mhealth-20-31 


\section{Introduction}

Despite the growing evidence linking inpatient sleep disturbance to detrimental outcomes such as delirium and falls (1-3), sleep deprivation and poor sleep quality are common in hospitalized patients (4). Unfortunately sleep has not been adequately assessed or managed in acute care hospitals (5). Limited understanding of the importance of sleep, lack of clear guidance of how to evaluate and improve sleep, and inadequate communication are all barriers to effective inpatient sleep management (5). Reflected by clinician's perceived lack of options to manage inpatient sleep, sedative-hypnotic medications are frequently prescribed for hospitalized patients, which can result in preventable harm including delirium, falls, hip fractures, and increased morbidity and mortality $(6,7)$. It is clear that addressing the underlying causes of poor sleep is a safer and more effective alternative to inpatient pharmacological sleep-aid use.

Studies testing non-pharmacologic sleep promotion interventions typically planned by clinicians to address one common inpatient sleep disruptor for all the patients (e.g., quiet time or white noise to minimize environmental noise, relaxation techniques such as music or massage to relieve anxiety), but this traditional "one-size-fits-all" strategy has demonstrated limited success (8). This is not surprising. For example, a study using white noise to improve sleep may not be beneficial if a portion of patients do not perceive noise as a disruptor to their sleep, or if some patients do not prefer white noise as a way to minimize noise. Going beyond this "one-size-fits-all" method, we believe that an individualized strategy with patient's input to identify what interferes their sleep and how to improve their sleep is more likely to succeed. As the first step making individualized sleep promotion feasible, we developed a brief assessment tool, the Factors Affecting Inpatient Sleep (FAIS) scale (9). The FAIS scale assesses 14 commons factors in three domains, including sleep interruptions due to hospital environment or medical care, sleep disturbance due to discomfort or care plan, and emotional or physical impairment due to illness or hospitalization. With established content validity and psychometric adequacy, the FAIS scale can be used on a daily basis to identify salient modifiable sleep disruptors in the hospital setting (9).

Our previous work demonstrates that routine assessment of sleep, open dialogue with patient, collaborative care planning, and tailored patient-centered interventions are key to successful inpatient sleep promotion (5).
Interventions using mobile health (mHealth) technologies, which improve timely communication, heighten patient involvement, and offer individual tailoring, hold great promise to address these key issues of sleep promotion $(10,11)$. As our continued effort of individualized sleep promotion, we have developed an innovative mHealth tool, Sleep Promotion Toolkit for hospitalized patients (SLEEPKit). With the FAIS scale built in as the basis of sleep assessment, the SLEEPKit aims to establish evidencebased linkages between routine assessment for modifiable sleep disruptors and tailored behavioral interventions incorporating patient's input to improve sleep in acute care hospitals. SLEEPKit uses icons to depict an easy-tointerpret summary of patient-specific sleep disruptors, and offers strategies targeting each sleep disruptor to support collaborative care planning. The objective of this report was to develop icons and strategies addressing each of the 14 common sleep disturbing factors on the FAIS scale, which were key components built into the SLEEPKit to guide real-time individualized sleep promotion.

\section{Methods}

\section{Overview}

A participatory iterative approach was used to develop and refine the icons and sleep promotion strategies for each of the 14 sleep disturbing factors on the FAIS scale. Feedbacks from stakeholders including patients, family members, and clinicians were obtained throughout the development process. Focus groups were conducted with patients/ family members and clinicians who served on the Patient/ Family Advisory Council, or the existing interdisciplinary professional practice committees at the study hospital. A semi-structured interview guide was used to focus and facilitate the interview process. Content analysis including data reduction, data display, and conclusion drawing were performed for each interview to derive topical codes to interpret the data (12). Institutional review board (IRB) approval at the study institute was obtained. All participants provided informed verbal consent.

\section{Developing and validating icons for sleep disturbing factors}

Focus groups were used to inform the initial development and to brainstorm for the refinement of the icons. Individual interviews with patients and clinicians were conducted to validate each version of the icons. The inclusion criteria 
for patient participants for the individual interviews were: admission to a patient care unit, awake and alert, cognitively intact, older than 18, able to understand and speak English to provide feedback on icons. Clinicians involved in direct patient care were invited for the interviews. Purposive sampling was used to ensure a diverse sample for patients and clinicians in terms of age, gender, race, education, and years of practice. During the individual interviews, participants rated their satisfaction of the icons using a standardized Content Validity Index (CVI) to see if the icon represented the concept on a 4-point Likert scale, with the choices of 1 (strongly disagree), 2 (moderately disagree), 3 (moderately agree), and 4 (strongly agree), and offered comments and suggestions for improvement (13). After reviewing CVI scores and feedback, the research team consulted with the illustrator to refine the icons. Successive phases of iterative icon evaluation and refinement were carried out until the average CVI ratings for each icon achieved 3 and above.

\section{Developing and refining strategies addressing sleep disturbing factors}

Strategies of sleep promotion were first identified by the literature review, and then enriched by the summary of data from our previous work, including interviewing data from patients and clinicians and written feedback collected from a survey $(5,9)$. Focus groups with clinicians and patients were conducted, in order to learn empirically if the sleep promotion tips on the current version were acceptable to both patients and clinicians, and how they could be improved. Inquiries were primarily focused on (I) if any important strategy was missing; (II) if the descriptions of the strategies were clear; and (III) if the strategies proposed to improve sleep were perceived to be effective and feasible in the acute care hospital setting.

\section{Results}

Six focus groups were conducted and achieved saturation in suggestions for improvement. Among them, three focus groups were conducted with patients and family members and nurses who served on the Patient/ Family Advisory Council, and three were conducted with nurses serving on the interdisciplinary professional practice committees. A total of 5 patients, 3 family members, and 42 nurses participated in the focus groups.
A total 75 patients and 50 clinicians (including 45 nurses 5 nurse assistants) offered individual feedback and the CVI test for the icons. Among the 75 patient participants, 56\% were female, $40 \%$ were over 65 years, $80 \%$ self-identified as non-Hispanic White, and $43 \%$ reported to have at least college education. The majority of the clinician participants were non-Hispanic White (86\%) females (92\%), 60\% of them with age ranged from 35 to 44, and with an average over 10 years of practice at current hospital. After two iterations, 12 out of the 14 icons achieved an average CVI score ranged from 3.3 to 4.0. Two additional rounds of iterations were conducted for the two icons "disturbed bedtime routine" and "reduced daytime physical activity" based on the feedback from individual interviews and focus group discussions, with their final versions achieved the mean CVI scores at or above 3.0. No significant differences were observed in the CVI scores between evaluations from patients and clinicians.

See http://fp.amegroups.cn/cms/1d112efcd3d0f8897cd 649c619ef1e19/mhealth-20-31-1.pdf lists the final version of icons and the sleep promotion tips targeting each sleep disturbing factor on the FAIS scale. The tips were created to demonstrate a collaborative effort between patients and clinicians. Based on the feedback from the focus groups, one challenge was that patients did not know how to share their concerns with the care team or how to make suggestions related to their care. To address this challenge and empower patients for bedside communication related to their sleep, we included examples of communication starters ("Try saying") in the tips for patients (see http:// fp.amegroups.cn/cms/1d112 efcd3d0f8897cd649c619ef 1e19/mhealth-20-31-1.pdf).

Figure 1 illustrates how the icons and sleep promotion tips were built into the SLEEPKit to support individualized sleep promotion. We include screen shots from the SLEEPKit app, using the sleep disturbing factor "worried about medical condition or procedures" as an example. Using SLEEPKit, the patient started a sleep self-assessment based on the previous night, including the 14 sleep disturbing factors using the FAIS scale. On the summary page for the sleep disturbing factors, the icons were highlighted if the patient responded "YES" to those factors. By clicking on the icon, the SLEEPKit offered strategies addressing this specific sleep disruptor for both patients and clinicians, with the goal to support bedside communication and collaborative care planning for sleep promotion. 

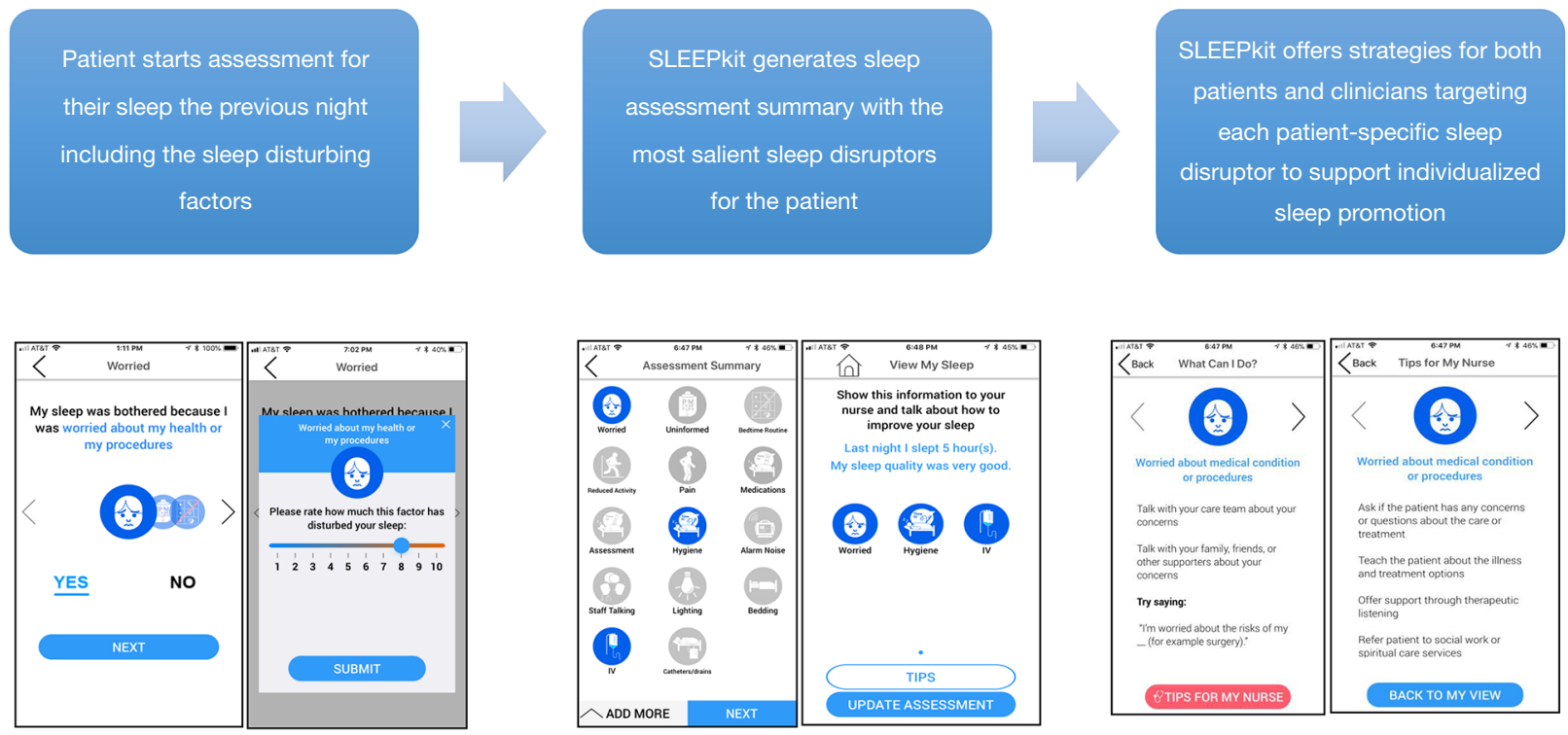

Figure 1 Workflow of the Sleep Promotion Toolkit (SLEEPKit) with screen shot examples.

\section{Discussion}

We developed a set of icons illustrating 14 common sleep disruptors and strategies to manage each of the sleep disturbing factors, which were built into the mHealth tool SLEEPKit to support individualized sleep promotion in acute care hospitals. This work is part of our effort to change current clinical practice related to patient sleep, and to advocate a new individualized sleep promotion strategy by addressing specific sleep disruptors and engaging patient in care planning and implementation. The set of icons and sleep promotion tips are part of the development of the SLEEPKit, which demonstrates the start of our endeavor of using mHealth technology to support individualized sleep promotion.

As we seek to improve care quality and safety through patient-centeredness, sleep as a basic human need which is critical to recovery should be emphasized, even in the context of complex and busy medical care (14). Inspired by the patient-centeredness, one strength of our work is the participatory approach engaging patients throughout the development process. The CVI testing technique, which was previously used to validate fall prevention icons supporting patient-centered education $(13,15)$, offers both quantitative and qualitative approaches maximizing user input in the icon development. By effectively illustrating common inpatient sleep disturbing factors, this set of icons can be used together with the FAIS scale as part of routine sleep assessment, as well as in both electronic and paper versions of education forms and clinical work sheets related to patient sleep.

The tips we proposed to address each sleep disruptor emphasize a collaborative effort and open dialogue between patient and clinician in order to achieve successful sleep promotion. Most of these sleep promotion strategies perceived as effective and feasible by both patients and clinicians are at low risk, low cost, and easy to implement. None of the interventions we propose involves radical redesign of the environmental space or care processes, which supports the feasibility of sleep promotion tailoring to individual patient's needs. However, clinicians could not know individual patient's preferences without asking (e.g., bedtime routine, preference of relaxation technique). This further emphasizes the need to communicate with patients and engage patients in the care planning process. The behavioral interventions of sleep promotion are still evolving, with overall insufficient to low strength of evidence supporting their effect to improve sleep quality or quantity for hospitalized patients (8). We acknowledge that the tips we proposed in http://fp.amegroups.cn/cms/1 d112efcd3d0f8897cd649c619ef1e19/mhealth-20-31-1.pdf reflect the current state of science and are perceived to be feasible in the study hospital, have not yet been systemically validated in practice; nevertheless, we propose these as a starting point. We anticipate future refinement of these tips 
to make them relevant to diverse patient populations and practice environments.

With routine sleep assessment including patient-specific sleep disturbing factors, open dialogue with patients, and collaborative care planning, we believe that individualized sleep promotion can be feasible and is likely to succeed in acute care hospitals. As a rapid growing area of research, mHealth interventions can be used to support these key features of successful sleep promotion. As we illustrated in Figure 1, the icons and sleep promotion tips we developed for each common inpatient sleep disruptor were built into the SLEEPKit, an mHealth tool to support individualized sleep promotion. The workflow of the SLEEPKit demonstrates the process of sleep assessment, identification of the salient modifiable sleep disruptors, and tailored education for both patients and clinicians on specific sleep disruptors to support bedside discussion and collaborative care planning. A separate report from our team will focus on our development and evaluation of the SLEEPKit from an implementation science perspective. As an innovative mHealth sleep promotion tool, we hope that SLEEPKit can be an exemplar supporting real-time tailored sleep management in home or long-term care settings.

\section{Conclusions}

Improving sleep for hospitalized patients is an essential clinical need. Patient-centered care is critically needed to improve inpatient sleep. Behavioral sleep promotion and minimization of pharmacological sleep-aid use continues to be part of the strategy that reduces delirium, hospital cost, and hospital length of stay (16). Sleep is known to be poor among hospitalized patients for many reasons. Compared to the traditional "one-size-fits-all" sleep promotion interventions designed by clinicians to address one or a few sleep disruptors delivered to all patients, an individualized strategy engaging patients to identify salient sleep disruptors and seek their input how to address these disruptors are more likely to succeed. Followed by our work of developing the Factors Affecting Inpatient Sleep (FAIS) scale to identify patient-specific sleep disruptors (9), in this report we developed a set of icons illustrating 14 common sleep disturbing factors on the FAIS scale, and proposed behavioral sleep promotion tips addressing each sleep disruptor for both patients and clinicians. By using the participatory iterative approach, these icons were understandable by both patients and clinicians, and the sleep promotion tips were perceived to be feasible and effective in the acute care hospital setting. This work moved the individualized sleep promotion forward, and supported the development of a novel mHealth tool SLEEPKit to support inpatient sleep promotion tailored to individual patient's needs.

\section{Acknowledgments}

The authors would like to thank the Graphic Designer, Ms. Wenpin Li, for her contribution to the icon illustration, and all the research assistants for their support in data collection and analysis. In addition, the authors thank all patients, family members, and clinicians from the Brigham and Women's Hospital who have offered their feedback to the development of icons and sleep promotion tips.

Funding: This study was funded by grant number R21HS024330 from the Agency for Healthcare Research and Quality. The content is solely the responsibility of the authors and does not necessarily represent the official views of the Agency for Healthcare Research and Quality.

\section{Footnote}

Provenance and Peer Review: This article was commissioned by the Guest Editor (Mei R Fu) for the series "Real-Time Detection and Management of Chronic Illnesses" published in $m$ Health. The article has undergone external peer review.

Conflicts of Interest: Both authors have completed the ICMJE uniform disclosure form. The authors have no conflicts of interest to declare (available at http://dx.doi.org/10.21037/ mhealth-20-31). The series "Real-Time Detection and Management of Chronic Illnesses" was commissioned by the editorial office without any funding or sponsorship. The authors have no other conflicts of interest to declare.

Ethical Statement: The authors are accountable for all aspects of the work in ensuring that questions related to the accuracy or integrity of any part of the work are appropriately investigated and resolved.

Open Access Statement: This is an Open Access article distributed in accordance with the Creative Commons Attribution-NonCommercial-NoDerivs 4.0 International License (CC BY-NC-ND 4.0), which permits the noncommercial replication and distribution of the article with the strict proviso that no changes or edits are made and the original work is properly cited (including links to both the 
formal publication through the relevant DOI and the license). See: https://creativecommons.org/licenses/by-nc-nd/4.0/.

\section{References}

1. Mazer SE. Increase patient safety by creating a quieter hospital environment. Biomed Instrum Technol 2006;40:145-6.

2. Patel M, Gomez S, Berg S, et al. Effects of 24-h and 36-h sleep deprivation on human postural control and adaptation. Exp Brain Res 2008;185:165-73.

3. Weinhouse GL, Schwab RJ, Watson PL, et al. Bench-tobedside review: delirium in ICU patients - importance of sleep deprivation. Crit Care 2009;13:234.

4. Wesselius HM, van den Ende ES, Alsma J, et al. Quality and Quantity of Sleep and Factors Associated With Sleep Disturbance in Hospitalized Patients. JAMA Intern Med 2018;178:1201-8.

5. Ye L, Keane K, Hutton Johnson S, et al. How do clinicians assess, communicate about, and manage patient sleep in the hospital? J Nurs Adm 2013;43:342-7.

6. Gillis CM, Poyant JO, Degrado JR, et al. Inpatient pharmacological sleep aid utilization is common at a tertiary medical center. J Hosp Med 2014;9:652-7.

7. Soong C, Burry L, Cho HJ, et al. An Implementation Guide to Promote Sleep and Reduce Sedative-Hypnotic Initiation for Noncritically Ill Inpatients. JAMA Intern Med 2019;179:965-72.

8. Tamrat R, Huynh-Le MP, Goyal M. Non-pharmacologic interventions to improve the sleep of hospitalized patients:

doi: $10.21037 /$ mhealth-20-31

Cite this article as: Ye L, Dykes PC. Individualized sleep promotion in acute care hospitals: managing specific factors that affect patient sleep. mHealth 2021;7:25. a systematic review. J Gen Intern Med 2014;29:788-95.

9. Ye L, Owens RL, Dykes P. Individualized sleep promotion in acute care hospitals: Identifying factors that affect patient sleep. Appl Nurs Res 2019;48:63-7.

10. Free C, Phillips G, Galli L, et al. The effectiveness of mobile-health technology-based health behaviour change or disease management interventions for health care consumers: a systematic review. PLoS Med 2013;10:e1001362.

11. Free C, Phillips G, Watson L, et al. The effectiveness of mobile-health technologies to improve health care service delivery processes: a systematic review and meta-analysis. PLoS Med 2013;10:e1001363.

12. Hsieh HF, Shannon SE. Three approaches to qualitative content analysis. Qual Health Res 2005;15:1277-88.

13. Leung WY, Adelman J, Bates DW, et al. Validating Fall Prevention Icons to Support Patient-Centered Education. J Patient Saf 2017. [Epub ahead of print].

14. Xu T, Wick EC, Makary MA. Sleep deprivation and starvation in hospitalised patients: how medical care can harm patients. BMJ Qual Saf 2016;25:311-4.

15. Hurley AC, Dykes PC, Carroll DL, et al. Fall TIP: validation of icons to communicate fall risk status and tailored interventions to prevent patient falls. Stud Health Technol Inform 2009;146:455-9.

16. Zaubler TS, Murphy K, Rizzuto L, et al. Quality improvement and cost savings with multicomponent delirium interventions: replication of the Hospital Elder Life Program in a community hospital. Psychosomatics 2013;54:219-26. 